\title{
Object Selection Based on Subclass Error Correcting for ALVOT
}

\author{
Miguel Angel Medina-Pérez ${ }^{1}$, Milton García-Borroto², \\ and José Ruiz-Shulcloper ${ }^{3}$ \\ ${ }^{1}$ University of Ciego de Ávila, Cuba \\ migue@bioplantas.cu \\ http: / / www. unica.cu \\ ${ }^{2}$ Bioplants Center, UNICA, C. de Ávila, Cuba \\ mil@bioplantas.cu \\ http: / /www.bioplantas.cu \\ ${ }^{3}$ Advanced Technologies Applications Center, MINBAS, Cuba \\ j.shulcloper@cenatav.co.cu \\ http: //www.cenatav.co.cu/
}

\begin{abstract}
ALVOT is a supervised classification model based on partial precedences. These classifiers work with databases having objects described simultaneously by numeric and nonnumeric features. In this paper a new object selection method based on the error per subclass is proposed for improving the accuracy, especially with noisy training matrixes. A comparative numerical experiment was performed with different methods of object selection. The experimental results show a good performance of the proposed method with respect to previously reported in the literature.
\end{abstract}

Keywords: Partial precedence, mixed and incomplete data, editing method.

\section{Introduction}

In general, the better the training matrix the higher the accuracy of supervised classifiers. The accuracy of a classifier depends on the way that it is measured, and in this paper it is directly proportional to the well classification ratio. On the other hand, the quality measure of the training matrix is considered as a function of the classifier accuracy. Improving the accuracy is usually accomplished by removing noisy as well as redundant objects. Several techniques have been developed to deal with these two problems: editing and condensing methods respectively [1].

In real world problems, sometimes the objects are described simultaneously in terms of numeric and nonnumeric features. Some of the object descriptions could also be incomplete (missing values). In these cases, we are talking about mixed and incomplete data (MID). For this kind of data, the tools of the Logical Combinatorial Pattern Recognition (LCPR) [2] have shown a good behavior.

ALVOT [3, 4], introduced by Zhuravlev, is a model of supervised classification based on partial precedence. Partial precedence is the principle of calculating the 
object similarities using comparisons between their partial descriptions. A partial description is a combination of feature values that should have a clear meaning in the problem domain. This is the way that physicians, and other natural scientists, establish comparisons among objects in practice [4]. This model of algorithms has been applied to different real world situations. In these cases new comparison criteria of feature values and similarity functions between sub-description of objects were introduced, allowing to work with MID.

Any algorithm of ALVOT model $A$ works in six stages:

Stage 1.- Determine the support sets system $\Omega_{A}$, which is a set of subsets of features Stage 2.- Find out the feature values comparison criteria and similarity function between sub-descriptions of objects $\beta\left(\Omega O, \Omega O_{i}\right), \Omega \in \Omega_{A} . \Omega O$ is a subdescription of object $O$ using only features in $\Omega$; and $\beta$ is a similarity function Stage 3.- Rule $\Gamma_{\Omega}\left(O_{i}, O\right)$ for evaluating the partial similarity between a fixed subdescription of a new object $O$ and the corresponding sub-descriptions of previously classified objects $O_{i}$ (partial evaluation by rows)

Stage 4.- Rule $\Gamma_{\Omega}^{j}(O)$ for summarizing the partial evaluation by rows of a fixed subdescription of a new object $O$ for class $K_{j}$ (partial evaluation by class)

Stage 5.- Rule $\Gamma^{j}(O)$ for summarizing all partial evaluations by class with respect to the whole support sets system of a new object $O$ (total evaluation by class)

Stage 6.- Decision-making rule $\Gamma_{A}(O)$ based on the total evaluation by class.

Some authors have faced the problem of improving the efficiency of an ALVOT classifier reducing the size of the training matrix. This is usually achieved with some negative impact in the classifier accuracy. In this paper a different approach is considered. We present an efficient method for improving the accuracy of the ALVOT classifiers.

\section{Previous Works}

The first editing method for ALVOT was introduced in [5, 6]. This method uses a set of genetic algorithms to select the support sets system, the features weights, and the objects in the training matrix respectively. Nevertheless, there are several problems where this optimization procedure can not be applied, because the parameters have a meaning in the problem definition domain. For example, the specialist might determine the support sets system and assign the feature weights according to his background knowledge. He can also use some procedures, with a comprehensive meaning in his model. Based on these facts, we compared our algorithm only with the object selection procedure (GA-ALVOT). 
The chromosomes used by the genetic algorithm were binary strings consisting of $m$ bits, one for each object, representing the subsets of those which are selected. The $i^{\text {th }}$ bit has value 1 when the respective element is included, and 0 otherwise.

In general, the algorithm works as follows:

1. The initial population is generated randomly. The population size and iteration number are input parameters of the algorithm.

2. The population's individuals are sorted according to their fitness. The first and last individuals are crossed, the second is crossed with the penultimate and this process is repeated until finishing the population. They are crossed using a 1-point crossover operator in the middle of the individual. The fitness function is the ratio of well classified objects.

3. For each individual in the population the mutation operator is applied. It takes randomly an individual's gene and changes its value. The fitness is evaluated for this new population.

4. The original individuals together with those obtained by crossing and mutation are sorted in descending order according to their fitness and those with highest fitness are chosen (taking into account the population size). The new population is used in the next iteration of the algorithm.

This method has an important random component, so two different executions of the algorithm with the same data could have dramatically different results. As pointed out by Kuncheva and Bezdek [7] selecting prototypes by Random Searches and Genetic Algorithms could be computationally demanding and, for large data sets, may be infeasible.

In the first numerical experiment of this paper our proposed method shows a clear superiority with respect to the GA-ALVOT. In the second experiment was considered larger training matrixes intentionally noised. Because the computational demanding of GA-ALVOT and its behavior in the first experiment, this procedure was not taken into consideration.

For selecting objects for ALVOT we can apply classical editing methods based on NN rule. An analogue solution was reported by Decaestecker [8] and Konig et. al [9], in which the training matrix is edited for a Radial Based Function network, using a procedure originally designed for k-NN. A deep study about editing methods is outside of the scope of this paper, but a review can be found in [1]. Considering we are working with MID, in this paper we use the Compact Set Editing method (CSE) [10].

Despite the fact that these procedures can be applied, as was shown in [11], a better solution could be achieved considering the way ALVOT works.

In [11] a method to select objects for ALVOT was introduced (Voting based Object Selection, VOS). In VOS the objects are selected according to their voting power in the training data, which is calculated with the equation (1).

$$
\imath(O)=\frac{V_{K_{j}}(O)}{\alpha_{K_{j}}(O)+1}-\max _{i \neq j}\left\{\frac{V_{K_{i}}^{\prime}(O)}{\alpha_{K_{i}}^{\prime}(O)+1}\right\}, \quad i \neq j
$$


where:

- $V_{K_{j}}(O)=\frac{1}{\left|K_{j}\right|-1} \sum_{\substack{O_{l} \in K_{j} \\ O_{l} \neq O}} I\left(O, O_{l}\right)$, summarizes the votes that $O \in K_{j}$ gives to the objects of the class $K_{j}$.

- $V_{K_{i}}^{\prime}(O)=\frac{1}{\left|K_{i}\right|} \sum_{O_{l} \in K_{i}} I\left(O, O_{l}\right)$, summarizes the votes that $O \in K_{j}$ gives to the objects of the class $K_{i}, i \neq j$.

- $I\left(O, O_{l}\right)=\left\{\begin{array}{cl}\frac{1}{\left|\Omega_{A}\right|} \sum_{\Omega \in \Omega_{A}} \frac{1}{\left|K_{j}\right|-1} \cdot \rho(O) \cdot \rho(\Omega) \cdot \beta\left(\Omega O, \Omega O_{l}\right) & \text { if } O_{l} \in K_{j} \\ \frac{1}{\left|\Omega_{A}\right|} \sum_{\Omega \in \Omega_{A}} \frac{1}{\left|K_{j}\right|} \cdot \rho(O) \cdot \rho(\Omega) \cdot \beta\left(\Omega O, \Omega O_{l}\right) & \text { if } O_{l} \in K_{i}\end{array} ;\right.$ a voting function obtained from ALVOT stages 3 to 5. This function expresses the vote that the object $O$ gives to $O_{l}$, being $\rho(O)$ the weight of the object $O$, $\rho(\Omega)=\sum_{\chi_{i} \in \Omega} \rho\left(\chi_{i}\right)$, and $\rho\left(\chi_{i}\right)$ the weight of the feature $\chi_{i}$.

- $\alpha_{K_{j}}(O)=\frac{1}{\left|K_{j}\right|-1} \sum_{\substack{O_{l} \in K_{j} \\ O \neq O_{l}}}\left|V_{K_{j}}(O)-I\left(O, O_{l}\right)\right|$; the deviation between the mean $V_{K_{j}}(O)$ and the vote that the object $O$ gives to the objects in $K_{j}$; so when the deviation decreases, the values of $l(O)$ increases.

- $\alpha_{K_{i}}^{\prime}(O)=\frac{1}{\left|K_{j}\right|} \sum_{O_{l} \in K_{i}}\left|V_{K_{i}}^{\prime}(O)-I\left(O, O_{l}\right)\right| ;$ the deviation between the mean $V_{K_{i}}^{\prime}(O)$ and the vote that the object $O$ gives to the objects in $K_{i}$; so when the deviation decreases, the values of $l(O)$ increases.

The expression (1) was modified with respect to [11]. Instead $\alpha_{K_{j}}(O)+\left(1-V_{K_{i}}(O)\right)$ and $\alpha_{K_{i}}^{\prime}(O)+\left(1-V_{K_{i}}^{\prime}(O)\right)$ here we use $\alpha_{K_{j}}(O)+1$ and $\alpha_{K_{i}}^{\prime}(O)+1$, respectively.

In general, the algorithm VOS works as follows:

- Calculate the voting power of each object using (1)

- Select an initial solution, formed by $\left.\min _{i \in 1 \ldots s}\left\{\left\{O \in K_{i}: \imath(O)>0\right\}\right\}\right\}$ objects with highest voting power per class, being $s$ the number of classes.

- Add (remove) the object per class with greater (lower) $l(O)$, until the original accuracy is at least (at most) achieved. 
In order to improve the results of VOS, in this paper we take into consideration the inner structure of the classes while selecting objects.

\section{The Inner Structure of the Classes in Supervised PR Problems}

In many classification problems, a class is not uniformly formed. This fact depends on the level of generality of the properties that determine each class. Consider, for example, in the universe of all humans we can define two classes: $S$ is the class of all who are sick, and $H$ is the class of all who are healthy. In the class $S$ are grouped together many different objects with many different diseases, which compose subclasses inside the outer class. Intuitively, if an object belongs to a subclass its most similar neighbor must be in the same set, so one way is to consider that a subclass should be a union of compact sets [10]. The compact set criterion induces a unique partition for a given data set, which has the property that one object $x$ and all its most similar neighbors belong to the same cluster, and also, those objects for which $x$ is its most similar neighbor.

Frequently in some compact sets there are objects with low similarity. Consequently, this structure is not appropriate for editing. That is why in this paper we introduce a new algorithm to generate the inner structure of the classes, named the Hierarchical Strongly Connected Components (HiSCC) algorithm. A strongly connected component of a direct graph is a maximal subset of vertices containing a directed path from each vertex to all others in the subset.

This algorithm assumes the following issues: a) The similarity between two components is computed as the global minimum similarity between two elements, one in each component (group-minimum); b) The maximum $\beta$-similarity graph is a direct graph where each vertex is connected to the vertexes with maximum value of similarity to it, if this value is above $\beta$. The algorithm works as follows:

Step 1: Construct the maximum $\beta$-similarity graph, $G_{0}$.

Step 2: Compute the set of all strongly connected components (SCC) of $G_{0}$, which are the initial solution $S C_{0}$.

Step 3: Construct the maximum $\beta$-similarity graph $G_{i}$, where the nodes are the elements of $S C_{i-1}$, and the inter-component similarity is the group-minimum.

Step 4: Find the strongly connected components of $G_{i}$ in the current iteration, $S C_{i}$

Step 5: If the number of elements in $S C_{i}$ and $S C_{i-1}$ differs, go to step 3.

\section{Object Selection Based on Subclass Error Correcting for ALVOT}

Based on the previous structuralization of the classes, a new method for editing ALVOT is introduced. Let $A$ be an algorithm of ALVOT and $T$ a training matrix formed by objects in $K_{1}, \ldots, K_{s}$ classes. 
Subclass Error Correcting Object Selection (SECOS) algorithm works as follows:

Step 1: Eliminate from $T$ all the objects with negative voting power, which are calculated with the equation (1). Let $T^{\prime}$ be the result matrix. (Noise filtering procedure)

Step 2: Cluster the matrix $T^{\prime}$ using HiSCC algorithm. Let $K_{i}^{a} \subseteq K_{i}, K_{j}^{b} \subseteq K_{j}$ where $i \neq j$ and $K_{i}^{a}, K_{j}^{b}$ are subclasses

Step 3: Build the validation matrix $V$, selecting the object with worst voting power from each cluster with cardinal greater than 1 .

Step 4: Build a confusion matrix $E_{A}$ of $A$. This is a square matrix where $E_{A}\left[K_{i}^{a}, K_{j}^{b}\right]$ represents the number of objects belonging to the cluster $K_{i}^{a} \subseteq K_{i}$; that were classified by $A$ as elements of $K_{j}^{b}$

Step 5: Select $K_{i}^{a}$ and $K_{j}^{b}$ with highest $E_{A}\left\lfloor K_{i}^{a}, K_{j}^{b}\right\rfloor, i \neq j$.

Step 6: Select $X=\left\{O \in K_{j}^{b}: V_{K_{i}^{a}}(O)>\frac{1}{\left|K_{i}^{a}\right|} \sum_{O^{\prime} \in K_{i}^{a}} V_{K_{i}^{a}}\left(O^{\prime}\right)\right\}$, and sort them descending according to $V_{K_{i}^{a}}(O)$, the summarization of the votes that $O$ gives to the objects of the subclass $K_{i}^{a}$. For every $O \in X$, classify the validation matrix $V$ using ALVOT trained with $T^{\prime} \backslash\{O\}$, until a better accuracy is achieved, and go to step 4. If no object $O$ exists or no improvement of the accuracy could be achieved, go to step 5 and select a different pair of subclasses

Step 7: If no new pair of subclasses $K_{i}^{a}$ and $K_{j}^{b}$ can be found in step 5, finish.

\section{Experimental Results}

The experiments were made using 7 databases from UCI [12] Repository of Machine Learning, with mixed and incomplete data. A description of these databases can be found in Table 1. We conducted two different experiments. The first one was the accuracy comparison of $A$ trained with the original matrix, and the edited matrix with GA-ALVOT, CSE, VOS and SECOS respectively. The results of this experiment are shown in table 2 and 3.

In the second experiment, we consider two databases. The training matrixes were intentionally noised interchanging the classes of fixed percent of objects. For example, in the Australian database we got from the respective training matrix $40 \%$ of the objects belonging to class 2 and put them in class 1 , and vice versa. With these training matrixes the algorithms ALVOT, VOS, CSE and SECOS were executed. In table 4 the results of this experiment are shown. 
Table 1. Databases used in the experiments

\begin{tabular}{lcccc}
\hline \multicolumn{1}{c}{ Database } & Objects & Features & Missing Values & Classes \\
\hline Breast-cancer & 286 & 9 & 9 & 2 \\
Breast-cancer-breast & 286 & 9 & 9 & 2 \\
Credit-screening & 690 & 15 & 37 & 2 \\
Credit-screening-A9 & 690 & 15 & 37 & 2 \\
Credit-screening-A12 & 690 & 15 & 37 & 2 \\
Hepatitis & 155 & 19 & 75 & 2 \\
Australian & 690 & 14 & 37 & 2 \\
\hline
\end{tabular}

Breast-cancer-breast is the same database as Breast-cancer, but with the feature breast used as class feature. Credit-screening-A9 (Credit-screening-A12) is the same database as Credit-screening, but with the feature A9 (A12) used as class feature.

Each database was split taking $70 \%$ for training and $30 \%$ for testing, repeating the process 5 times and averaging the results.

In our experiments we used a voting algorithm $A$ with the following parameters:

- The support sets system was the set of all typical testors of the training matrix.

- $\beta\left(\Omega O_{i}, \Omega O_{j}\right)=\frac{\sum_{\chi_{p} \in \Omega} C_{p}\left(\chi_{p}\left(O_{i}\right), \chi_{p}\left(O_{j}\right)\right)}{|\Omega|}$; partial similarity function, where

$\Omega$ is a subset of features, $C_{p}$ the comparison criterion for the feature $\chi_{p}$, and $\chi_{p}\left(O_{i}\right)$ is the value of the feature $\chi_{p}$ in the object $O_{i}$.

- $C_{p}\left(\chi_{p}\left(O_{1}\right), \chi_{p}\left(O_{2}\right)\right)=\left\{\begin{array}{cc}1 & \text { if }\left|\chi_{p}\left(O_{1}\right)-\chi_{p}\left(O_{2}\right)\right|<\sigma_{p} \text {;similarity compare- } \\ 0 & \text { otherwise }\end{array}\right.$ son criteria for numeric features, where $\sigma_{p}$ is the standard deviation of the values of the feature $\chi_{p}$ in the training matrix.

- $C_{p}\left(\chi_{p}\left(O_{1}\right), \chi_{p}\left(O_{2}\right)\right)=\left\{\begin{array}{cc}1 & \text { if } \chi_{p}\left(O_{1}\right)=\chi_{p}\left(O_{2}\right) \\ 0 & \text { otherwise }\end{array} ;\right.$ similarity comparison criteria for non-numeric features.

- $\Gamma_{\Omega}\left(O_{p}, O\right)=\rho\left(O_{p}\right) \cdot \rho(\Omega) \cdot \beta\left(\Omega O_{p}, \Omega O\right)$; partial evaluation by rows for a fixed support set, where $\rho(\Omega)=\sum_{\chi_{i} \in \Omega} \rho\left(\chi_{i}\right)$. In the experiments $\rho\left(O_{p}\right)=1$ was used.

- The weight of the feature $\chi_{i}$ is calculated, following [13], with the equation: $\rho\left(\chi_{i}\right)=\alpha \cdot P\left(\chi_{i}\right)+\beta \cdot L\left(\chi_{i}\right)$ with $\alpha, \beta>0$ and $\alpha+\beta=1$, being $\alpha$ and 
$\beta$ two parameters, which weight the influence of $P\left(\chi_{i}\right)$ and $L\left(\chi_{i}\right)$ respectively in $\rho\left(\chi_{i}\right)$. We used $\alpha=\beta=0.5 . P\left(\chi_{i}\right)$ is computed: $P\left(\chi_{i}\right)=\frac{\tau(i)}{\tau}$, where $\tau(i)$ is the number of typical testors, which contain the feature $\chi_{i}$ and $\tau$ the amount of all typical testors. $L\left(\chi_{i}\right)$ is computed: $L\left(\chi_{i}\right)=\frac{\sum_{t \in \Psi^{*}\left(\chi_{i}\right)} \frac{1}{|t|}}{\left|\Psi^{*}\left(\chi_{i}\right)\right|}$, where $\Psi^{*}\left(\chi_{i}\right)$ is the family of all typical testors, which contain the feature $\chi_{i}$

- $\Gamma_{\Omega}^{j}(O)=\frac{1}{\left|K_{j}\right|} \sum_{O_{t} \in K_{j}} \Gamma_{\Omega}(O, O)$; partial evaluation by classes for a fixed support set

- $\Gamma^{j}(O)=\frac{1}{\left|\Omega_{A}\right|} \sum_{\Omega \in \Omega_{A}} \Gamma_{\Omega}^{j}(O)$; total evaluation by classes

- $\Gamma_{A}\left(\Gamma^{1}(O), \ldots, \Gamma^{s}(O)\right)$; the majority vote.

The quality function used was the well classification ratio.

The accuracy of the original ALVOT $A$, GA-ALVOT, VOS, CSE, and SECOS with the edited matrixes respectively, is shown in Table 2. The accuracy of $A$ could be improved in 3 cases. In 2 of them, SECOS achieved the highest value.

The compression ratios achieved by the algorithms are shown in Table 3 .

Table 2. Accuracy of $A$ without editing compared with edited by respective methods

\begin{tabular}{lllccr}
\hline \multicolumn{1}{c}{ Database } & $A$ & GA-ALVOT & CSE & VOS & SECOS \\
\hline Breast-cancer & 0.75 & 0.71 & 0.75 & 0.76 & $\mathbf{0 . 7 8}$ \\
Breast-cancer-breast & $\underline{\mathbf{0 . 5 8}}$ & 0.52 & 0.57 & 0.57 & 0.57 \\
Credit-screening & $\underline{\mathbf{0 . 8 1}}$ & 0.79 & 0.79 & 0.79 & 0.79 \\
Credit-screening-A9 & $\overline{0.73}$ & $\underline{\mathbf{0 . 7 4}}$ & $\underline{\mathbf{0 . 7 4}}$ & 0.71 & 0.72 \\
Credit-screening-A12 & $\underline{\mathbf{0 . 6 4}}$ & 0.62 & 0.61 & 0.62 & 0.62 \\
Hepatitis & 0.73 & 0.74 & 0.68 & 0.75 & $\mathbf{0 . 7 8}$ \\
\hline
\end{tabular}

The performance of each editing method in the presence of noisy databases is shown in table 4. Because of the stability of the ALVOT classifier, its accuracy can not be degraded with low levels of noise. Nevertheless if the noise introduced is too much, the degradation is drastic, and no meaningful comparison can be done. That is why we only present the results for noise of $30 \%$ and $40 \%$. The results show that SECOS is less sensitive to noise than other editing methods, and also it improves original ALVOT. This behavior can be explained because it starts with a noise filtering procedure. 
The CSE editing method, although with acceptable behavior in experiment 1, has worse results in the presence of noise. We suppose this happens because CSE does not select objects taking into consideration the way ALVOT works.

Table 3. Compression ratio

\begin{tabular}{lcccc}
\hline \multicolumn{1}{c}{ Database } & GA-ALVOT & CSE & VOS & SECOS \\
\hline Breast-cancer & $\underline{\mathbf{0 . 5 1}}$ & 0.41 & 0.35 & 0.41 \\
Breast-cancer-breast & $\underline{\mathbf{0 . 5 3}}$ & 0.36 & 0.36 & 0.46 \\
Credit-screening & 0.50 & $\underline{\mathbf{0 . 5 2}}$ & 0.51 & 0.20 \\
Credit-screening-A9 & $\underline{\mathbf{0 . 5 0}}$ & 0.48 & 0.26 & 0.21 \\
Credit-screening-A12 & 0.49 & 0.38 & 0.23 & $\underline{\mathbf{0 . 5 1}}$ \\
Hepatitis & 0.51 & 0.54 & $\underline{\mathbf{0 . 6 5}}$ & 0.32 \\
\hline
\end{tabular}

Table 4. Accuracy of $A$ with larger and noised training matrixes

\begin{tabular}{lllll}
\hline \multicolumn{1}{c}{ Database/noise } & $A$ & VOS & CSE & SECOS \\
\hline Australian & 0.85 & & & \\
Australian $/ 30 \%$ & 0.84 & $\underline{\mathbf{0 . 8 5}}$ & $\underline{\mathbf{0 . 8 5}}$ & $\underline{\mathbf{0 . 8 5}}$ \\
Australian/40\% & 0.82 & 0.81 & 0.70 & $\underline{\mathbf{0 . 8 4}}$ \\
Credit-screening & 0.86 & & & \\
Credit-screening/30\% & $\underline{\mathbf{0 . 8 6}}$ & 0.85 & 0.85 & $\underline{\mathbf{0 . 8 6}}$ \\
Credit-screening/40\% & 0.81 & 0.81 & 0.75 & $\underline{\mathbf{0 . 8 2}}$ \\
\hline
\end{tabular}

\section{Conclusions}

ALVOT is a model for supervised classification based on partial precedences. It is mainly used in problems where the objects are described in terms of numeric and nonnumeric features simultaneously with missing values.

Speeding up ALVOT through object selection has been faced in some papers, but it is usually achieved with some degradation of the classifier accuracy. In this paper a method for improving the accuracy of ALVOT is presented: Subclass Error Correcting Object Selection (SECOS).

SECOS first eliminates noisy objects using the voting power concept. Then it decomposes each class in subclasses by HiSCC, a new subclass detecting algorithm. Finally, a confusion matrix by subclasses is used to select the objects to be eliminated.

Two different experiments were carried out. In the first, the comparisons with other methods showed that only in 3 databases of 6 the ALVOT accuracy could be improved. In 2 of them, SECOS achieved the highest value. In the second experiment artificial disturbance was introduced and it showed that the accuracy of ALVOT could be affected by noisy objects. The proposed algorithm improved the accuracy of the classifier in all databases so it can be used to detect noisy objects and improve the accuracy of ALVOT. 


\section{References}

1. Bezdek, J.C., Kuncheva, L.I.: Nearest Prototype classifiers designs: an experimental study. International Journal of Intelligent Systems 16, 1445-1473 (2001)

2. Martínez Trinidad, J.F., Guzmán-Arenas, A.: The logical combinatorial approach to Pattern Recognition, an overview through selected works. Pattern Recognition 34, 741$751(2001)$

3. Zhuravlev, Y.I., Nikiforov, V.V.: Recognition algorithms based on voting calculation. Journal Kibernetika 3, 1-11 (1971)

4. Ruiz-Shulcloper, J., Abidi, M.A.: Logical Combinatorial Pattern Recognition: A Review. In: Pandalai, S.G. (ed.) Recent Research Developments in Pattern Recognition. Transword Research Networks, USA, pp. 133-176 (2002)

5. Carrasco-Ochoa, J.A., Martínez-Trinidad, J.F.: Editing and Training for ALVOT, an Evolutionary Approach. In: Liu, J., Cheung, Y.-m., Yin, H. (eds.) IDEAL 2003. LNCS, vol. 2690, pp. 452-456. Springer, Heidelberg (2003)

6. Carrasco-Ochoa, J.A., Martínez-Trinidad, J.F.: Combining Evolutionary Techniques to Improve ALVOT Efficiency. WSEAS Transactions on Systems 2, 1073-1078 (2003)

7. Kuncheva, L.I., Bezdek, J.C.: Nearest prototype classification: clustering, genetic algorithms or random search. IEEE Transactions on Systems, Man and Cybernetics. Part C 28, 160-164 (1998)

8. Decaestecker, C.: NNP: A neural net classifier using prototype. International Conference on Neural Networks, San Francisco, California, pp. 822-824 (1993)

9. König, A., Rashhofer, R.J., Glesner, M.: A novel method for the design of radial-basisfunction networks and its implication for knowledge extraction. International Conference on Neural Networks, Orlando, Florida 1804-1809 (1994)

10. García-Borroto, M., Ruiz-Shulcloper, J.: Selecting Prototypes in Mixed Incomplete Data. In: Sanfeliu, A., Cortés, M.L. (eds.) CIARP 2005. LNCS, vol. 3773, pp. 450-459. Springer, Heidelberg (2005)

11. Medina-Perez, M.A., García-Borroto, M., Villuendas-Rey, Y., Ruiz-Shulcloper, J.: Selecting objects for ALVOT. In: Martínez-Trinidad, J.F., Carrasco Ochoa, J.A., Kittler, J. (eds.) CIARP 2006. LNCS, vol. 4225, pp. 606-613. Springer, Heidelberg (2006)

12. Merz, C.J., Murphy, P.M.: UCI Repository of Machine Learning Databases. University of California at Irvine, Department of Information and Computer Science, Irvine (1998)

13. Lazo-Cortés, M., Ruiz-Shulcloper, J.: Determining the feature informational weight for non-classical described objects and new algorithm to calculate fuzzy testors. Pattern Recognition Letters 16, 1259-1265 (1995) 